\title{
Scalar model of flocking dynamics on complex social networks
}

\author{
M.-Carmen Miguel ${ }^{1,2}$ and Romualdo Pastor-Satorras $\oplus^{3}$ \\ ${ }^{1}$ Departament de Física de la Matèria Condensada, Universitat de Barcelona, Martí i Franquès 1, 08028 Barcelona, Spain \\ ${ }^{2}$ Universitat de Barcelona Institute of Complex Systems (UBICS), Universitat de Barcelona, 08028 Barcelona, Spain \\ ${ }^{3}$ Departament de Física, Universitat Politècnica de Catalunya, Campus Nord B4, 08034 Barcelona, Spain
}

(Received 27 July 2019; published 14 October 2019)

\begin{abstract}
We investigate the effects of long-range social interactions in flocking dynamics by studying the dynamics of a scalar model of collective motion embedded in a complex network representing a pattern of social interactions, as observed in several social species. In this scalar model we find a phenomenology analogous to that observed in the classic Vicsek model: In networks with low heterogeneity, a phase transition separates an ordered from a disordered phase. At high levels of heterogeneity, instead, the transition is suppressed, and the system is always ordered. This observation is backed up analytically by the solution of a modified scalar model within an heterogeneous mean-field approximation. Our work extends the understanding of the effects of social interactions in flocking dynamics and opens the path to the analytical study of more complex topologies of social ties.
\end{abstract}

DOI: 10.1103/PhysRevE.100.042305

\section{INTRODUCTION}

The collective motion of interacting mobile agents can lead to stunning self-organized spatiotemporal patterns, such as those observed in flocks of birds, shoals of fish, or herds of mammals at the macroscopic scale, or in colonies of bacteria, migrating cells, or self-propelled nanoparticles at the microscopic scale [1-3]. The study of collective motion, and in particular the flocking behavior of animals, started attracting interest decades ago, the first studies dating from 1987, when Reynolds simulated the flocking of birds in terms of an artificial life simulation using boids [4]. The interest for this phenomenology in the statistical physics community ignited with the flocking model introduced by Vicsek and coworkers [5] in 1995. In the so-called Vicsek model, self-propelling particles (SPPs) move in a $d=2$ space with constant speed and interact among them by aligning their velocity to the average velocity of a set of other SPPs in their close neighborhood. The addition of a source of noise $\eta$, accounting for the physical difficulties in gathering and/or processing local information, leads to a dynamic phase transition separating an ordered phase at $\eta \leqslant \eta_{c}$, in which particles move coherently in the same average direction, from a disordered phase at $\eta>\eta_{c}$, in which SPPs move essentially as uncorrelated persistent random walkers [6]. The Vicsek model has allowed researchers to draw useful analogies between the collective motion of animals $[7,8]$ and the well-known features of order-disorder phase transitions in classical statistical mechanics [1,9].

The analysis of the Vicsek and other flocking models has been performed mostly in Euclidean spaces [9], where the neighborhood of a SPP is defined in a metric way, given by all other SPPs within a distance $R$ centered at the original SPP. Other works have also considered nonmetric neighborhoods, given by the SPPs in the first shell in a Voronoi tessellation constructed from the position of the particles at each time step [10]. This sort of interaction assumes that all individuals (SPPs) are equivalent and therefore simplify numerical and analytical approximations [11]. This simplification, however, comes at the cost of disregarding the possible effects of social interactions, which can cause individuals to follow preferentially those others with which they have strong social ties [12]. The effects of such social ties, represented in terms of nonmetric pairwise interactions encoded in a complex network [13], have been considered in the framework of the Vicsek model in different works [14-17]. In particular, Ref. [17] studied the effects of a heterogeneous complex topology, as represented by a scale-free degree distribution (defined as the probability that a node is connected to $k$ others or has degree $k$ ) with a power-law form [18], $P(k) \sim k^{-\gamma}$, on the order-disorder transition experimented by the model. In this work, it was reported that for $\gamma>5 / 2$, a standard transition is observed for a finite value of $\eta$, while for $\gamma<$ $5 / 2$, the transition is suppressed in the thermodynamic limit of infinite network size, being the system in the ordered state for all physical values of the noise parameter $\eta{ }^{1}$ This result is relevant for the understanding of the flocking behavior of a variety of social animals whose social contact networks [19] have been reported to have scale-free signatures [20,21], and it indicates that flocking is more robust against external fluctuations in the case of high network heterogeneity (i.e., small $\gamma$ ).

The results presented in Ref. [17] were backed up by numerical simulations and argued to be related with the behavior observed analytically in the majority-vote (MV) model in networks [22-25]. In the MV model, nodes are endowed with binary spin variables, taking values $\{+1,-1\}$. With probability $1-f$, nodes copy the spin orientation of the majority

\footnotetext{
${ }^{1}$ In the standard Vicsek model, the range of physical values of $\eta$ are restricted to a finite interval that can be taken to be $0 \leqslant \eta \leqslant 1$ [5]. In Ref. [17] it was shown that $\eta_{c} \rightarrow 1$ in the thermodynamic limit for $\gamma<5 / 2$.
} 
of their neighbors, while with probability $f$ they adopt the orientation opposite to that of the majority. A dynamic phase transition is observed in the MV at a noise threshold $f_{c}$, separating an ordered phase for $f<f_{c}$ from a disordered one at $f>f_{c}$. Analytical calculations on scale-free networks, based on a heterogeneous mean-field (HMF) theory [26,27], provide the expression of the threshold [28]

$$
f_{c}=\frac{1}{2}-\frac{1}{2} \sqrt{\frac{\pi}{2}} \frac{\langle k\rangle}{\left\langle k^{3 / 2}\right\rangle},
$$

where $\left\langle k^{n}\right\rangle=\sum_{k} k^{n} P(k)$ is the $n$th moment of the degree distribution. In an infinite network, this formula implies that for $\gamma>5 / 2$ a threshold $f_{c}<1 / 2$ is obtained, while for $\gamma<5 / 2$, the noise threshold takes its maximum value $f_{c}=$ $1 / 2$. This value, corresponding to a completely disordered system, indicates that the system is always ordered in the thermodynamic limit. The behavior of the MV model is thus akin to that observed in the Vicsek model on networks, and one can argue that they are equivalent in the sense that the dimensionality of the order parameter appears to be irrelevant in the characterization of the behavior of critical transitions in networks [17,27].

In this paper we delve further into the role of a complex topology in collective motion by considering the model of flocking dynamics proposed by Czirók, Barabási, and Vicsek (CBV) [29]. In the CBV model particles move in a onedimensional ring with a velocity represented by a real number, $u_{i} \in \mathbb{R}$, which tends to align with the average velocity of other particles in a local neighborhood, and which is affected by a random noise of amplitude $\eta$. This model, characterized by a continuous scalar order parameter, has been used to model the marching behavior of swarms of locusts [30,31]. Numerical simulations as well as analytical calculations based on a continuous hydrodynamic description show that the CBV model in one dimension experiences a dynamic phase transition, separating an ordered phase at low noise from a disordered one at high noise. The observed nonequilibrium phase transition is characterized by a set of exponents different from those observed in the vectorial standard Vicsek model in $d=2$ [29]. Here we show, by means of extensive numerical simulations, that a complex topology affects the CBV model in a way analogous to the Vicsek model: In scale-free topologies with degree exponent $\gamma>5 / 2$, a standard transition is preserved; on the other hand, for $\gamma<5 / 2$, the transition is absent in the thermodynamic limit. The two models, however, show differences in the critical exponents computed at the transition point. Our numerical analysis is complemented by the analytical solution within the HMF approximation of a modification of the CBV model, showing that the critical noise in the thermodynamic limit is proportional to the moment $\left\langle k^{3 / 2}\right\rangle$ of the degree distribution and thus drastically changing its behavior when $\gamma$ crosses the value 5/2. The HMF approximation allows us to compute the critical exponent $\beta$ in the ordered phase, which is found to be different from the one obtained for the MV model. Our results extend the range of analyses of collective motion models on networks and suggest that the presence of the crossover degree exponent $\gamma=5 / 2$ might be a general feature of flocking models on networks based on averaging rules applied to nearest neighbors.

\section{THE CBV MODEL IN NETWORKS}

The CBV model [29] was originally defined on a $d=1$ space with periodic boundary conditions, in which particles can move with velocity $u_{i}$. Each particle $i$ updates its velocity by taking the average $\langle u\rangle_{i}$ of the velocity of other particles in a neighborhood $\mathcal{V}_{i}=\left[x_{i}-\Delta, x_{i}+\Delta\right]$ surrounding it, i.e., $\langle u\rangle_{i}=\sum_{j \mid x_{j} \in \mathcal{V}_{i}} u_{j} / N_{i}$, where $N_{i}$ in the number of particles in $\mathcal{V}_{i}$. The average velocity is modulated by a function $G(u)$, such that, when $u>1, G(u)<u$, and when $u<1, G(u)>u$, and symmetrically for negative velocities, in such a way to force the modulus of the velocities to stay close to 1 . The function used in Ref. [29] is

$$
G(u)=\frac{u+\operatorname{sgn}(u)}{2},
$$

where $\operatorname{sgn}(u)$ is the sign function. Finally, a noise term $\eta \xi_{i}$ is added, where $\eta$ gauges the noise strength and $\xi_{i}$ is a random number uniformly distributed in the interval $[-1 / 2,1 / 2]$.

In the case of interactions mediated by a network, the topology is defined by the adjacency matrix $a_{i j}$, taking value 1 if nodes $i$ and $j$ are connected by an edge and zero otherwise [13]. We define the dynamical update rule for velocities as

$$
u_{i}(t+1)=G\left[\frac{\sum_{j} a_{i j} u_{j}(t)}{k_{i}}\right]+\eta \xi_{i} .
$$

With this prescription, we do not consider the interaction of a node with itself. This particular choice does not have a strong effect on the dynamics of the model, with the exception of possibly reducing the critical value of the noise. Obviously, the prescription presents problems for nodes of degree 1 , which simply consider their only neighbor. To avoid this problem, in the following we will consider a sufficiently large minimum degree. As in the case of the vectorial Vicsek model on networks, the position of particles plays no role, and we do not keep track of them [17].

The order parameter of the model, $\phi(\eta)$ is defined in terms of the time average of the average velocity

$$
\phi_{\eta}(t)=\frac{1}{N} \sum_{i} u_{i}(t)
$$

namely,

$$
\phi(\eta)=\left\langle\phi_{\eta}(t)\right\rangle_{t} \equiv \lim _{T \rightarrow \infty} \frac{1}{T} \int_{0}^{T} \phi_{\eta}(\tau) d \tau .
$$

In this model we expect the presence of a critical point $\eta_{c}$ separating a disordered phase with $\phi(\eta)=0$ for $\eta>\eta_{c}$ from an ordered or flocking one at $\eta<\eta_{c}$, in which $\phi(\eta) \sim\left(\eta_{c}-\right.$ $\eta)^{\beta}$, defining the critical exponent $\beta$.

\section{NUMERICAL ANALYSIS}

In order to check the behavior of the CVB model, we have performed extensive numerical simulations on uncorrelated power-law networks with degree distribution $P(k) \sim k^{-\gamma}$ and minimum degree $m=3$, generated using the uncorrelated configuration model (UCM) [32]. To estimate the order parameter we perform averages over 250000 time steps, after letting the dynamics thermalize for a sufficiently large time. Due to the velocity reversal invariance of the dynamics, we 

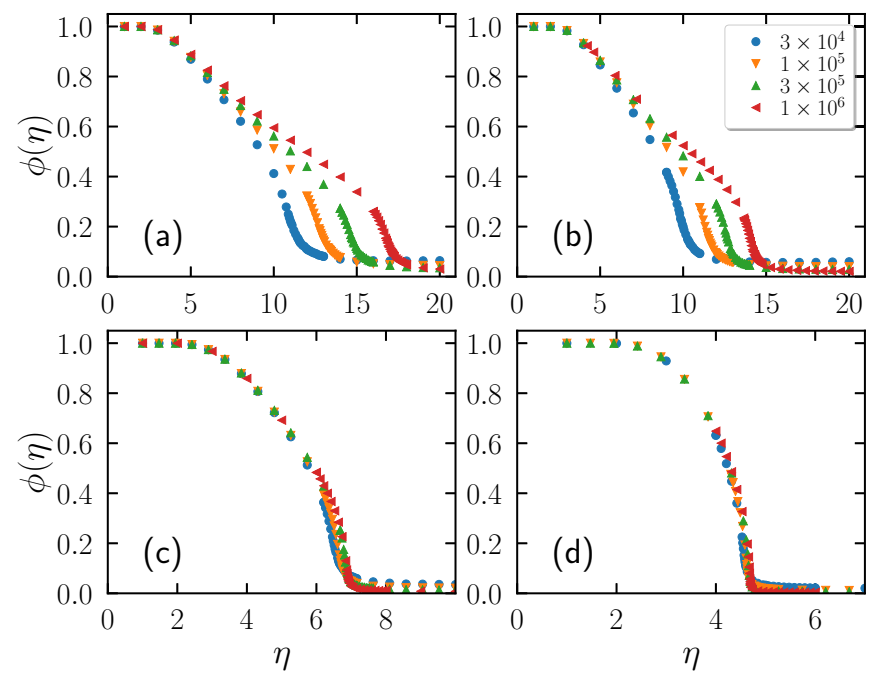

FIG. 1. Order parameter $\phi(\eta)$ as a function of $\eta$ in the CBV model on UCM networks of different size $N$. Panels correspond to different values of the degree exponent: (a) $\gamma=2.10$, (b) $\gamma=2.20$, (c) $\gamma=2.75$, (d) $\gamma=3.50$.

actually compute the order parameter as the time average of the absolute value of average velocity, $\phi(\eta)=\left\langle\left|\phi_{\eta}(t)\right|\right\rangle_{t}$.

In Fig. 1 we show the order parameter $\phi(\eta)$ as a function of the noise intensity $\eta$ computed in networks of different degree exponent $\gamma$ and size $N$. As we can see from this figure, in the region $\gamma>5 / 2$ the curves $\phi(\eta)$ seem to collapse for a sufficiently large network size, while for $\gamma<5 / 2$ the value of $\eta$ at which the order parameter becomes zero increases with $N$. This behavior is fully compatible with the observations made for the vectorial Vicsek model. One difference stands out, however. In the definition of the Vicsek model, the maximum physical value of the noise is $\eta=1$. In the CBV model, instead, $\eta$ is unbounded, so we expect the critical noise $\eta_{c}$ to diverge with network size for $\gamma<5 / 2$.

In order to further check this expectation, we have computed the dynamic susceptibility [17,33,34]:

$$
\chi_{N}(\eta)=N \frac{\left\langle\phi_{\eta}(t)^{2}\right\rangle_{t}-\phi(\eta)^{2}}{\phi(\eta)} .
$$

In the presence of a second-order phase transition, the dynamic susceptibility develops a peak at a value $\eta_{c}(N)$ that is interpreted as the size-dependent critical point in a network of fixed size $N$. In Fig. 2 we plot the dynamic susceptibility computed from different $\gamma$ and $N$ values. For all values of $\gamma$ considered, the dynamic susceptibility shows the presence of a sharp peak, signaling the corresponding finite-size critical point that for $\gamma>5 / 2$ tends to a constant value, while it diverges for $\gamma<5 / 2$.

In Fig. 3 we plot the value of the effective critical point $\eta_{c}(N)$ estimated from the peak of the susceptibility as a function of the network size $N$. For $\gamma>5 / 2$, the peaks tend to a constant value for increasing $N$. We plot as dashed lines the critical point in the thermodynamic limit, $\eta_{c}(\infty)$, estimated by applying a finite-size scaling of the form $\eta_{c}(N)=\eta_{c}(\infty)-$ $a N^{-b}$ [35]. The values estimated by performing a nonlinear regression of the numerical data to this ansatz form are $\eta_{c}(\infty)=$ 6.91(1) for $\gamma=2.75, \eta_{c}(\infty)=5.79(1)$ for $\gamma=3.00$, and
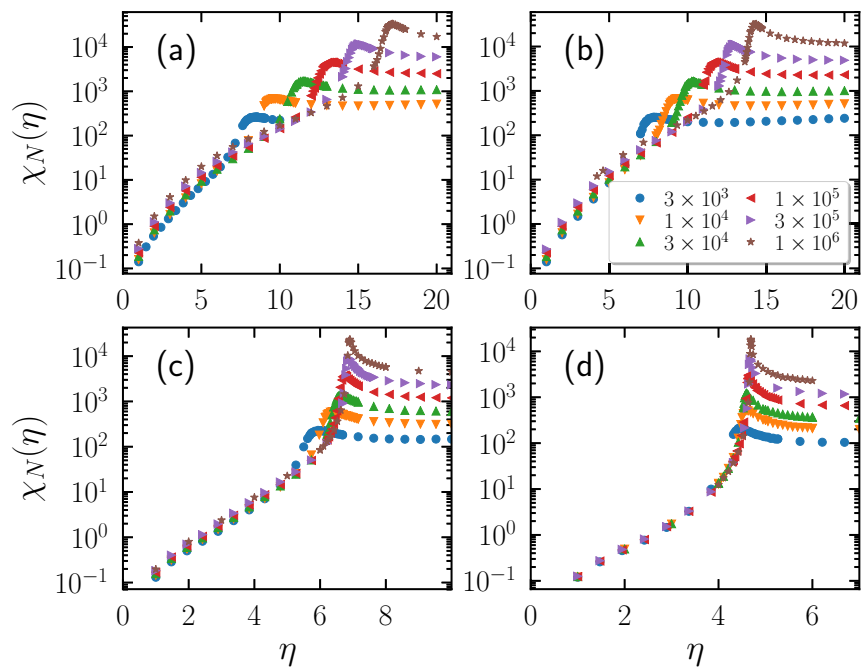

FIG. 2. Dynamic susceptibility $\chi_{N}(\eta)$ as a function of $\eta$ in the CBV model on UCM networks of different size $N$. Panels correspond to different values of the degree exponent: (a) $\gamma=2.10$, (b) $\gamma=$ 2.20 , (c) $\gamma=2.75$, (d) $\gamma=3.50$.

$\eta_{c}(\infty)=4.70(1)$ for $\gamma=3.50$, which are compatible with the peaks obtained for the largest size $N=10^{6}$ considered. For $\gamma<5 / 2$, on the other hand, the effective thresholds seems to grow as a power law with $N$. A linear regression to the form $\eta_{c}(N) \sim N^{b}$, performed over the four largest network sizes, leads to the exponent $b=0.11$ for $\gamma=2.10$ and $b=0.09$ for $\gamma=2.20$. The effective critical point $\eta_{c}(N)$ growing as a power law of the network size indicates an infinite critical noise in the thermodynamic limit, fully compatible with the maximal noise $\eta_{c}=1$ in the thermodynamic limit obtained in the Vicsek model.

Another signature of criticality in the order-disorder transition of the CBV model on networks is given by the height of the peak of the dynamic susceptibility $\chi^{\text {peak }}(N)$, which is

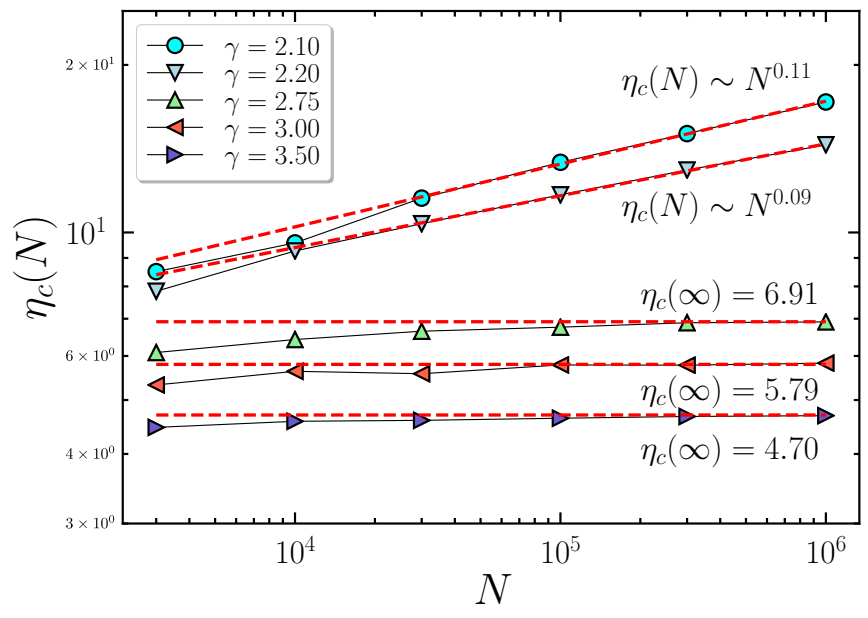

FIG. 3. Peak of the dynamic susceptibility $\eta_{c}(N)$ as a function of network size $N$ in the CBV model on UCM networks of different degree exponent $\gamma$. Dashed lines represent the critical point at the thermodynamic limit estimated using a finite-size scaling ansatz $(\gamma>5 / 2)$ and a power-law fit to the last four points $(\gamma<5 / 2)$. 


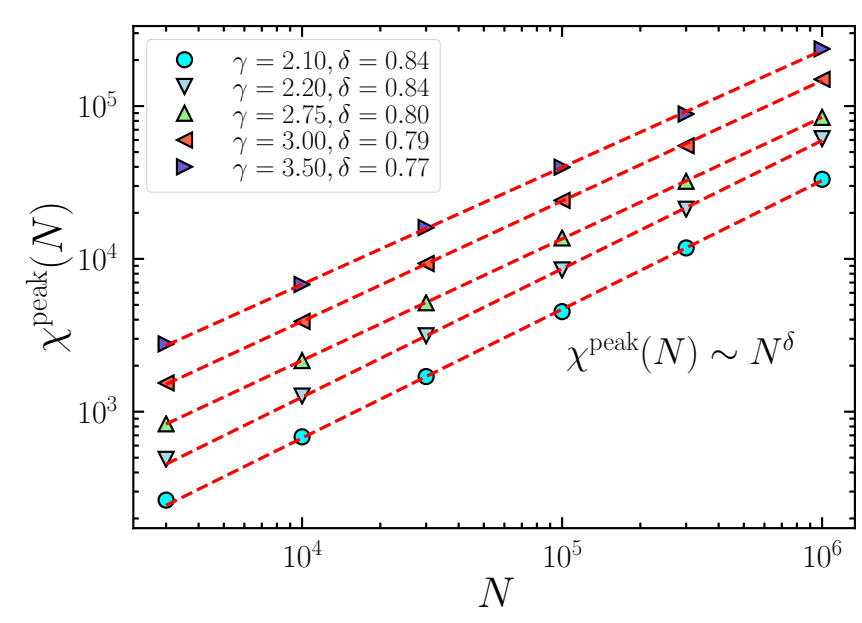

FIG. 4. Scaling of the peak of the dynamic susceptibility $\chi^{\text {peak }}(N)$ as a function of network size $N$ in the CBV model on UCM networks of different degree exponent $\gamma$. The exponents $\delta$ quoted are obtained by means of a linear regression to the form $\chi^{\text {peak }}(N) \sim N^{\delta}$. Plots have been shifted vertically for the sake of clarity.

expected to scale as a power law of the network size, $\chi^{\text {peak }}(N) \sim N^{\delta}[33,34]$, where $\delta$ is another characteristic critical exponent. In Ref. [17] it was observed numerically that the exponent $\delta$ for the vectorial Vicsek model on scale-free networks takes, as a function of $\gamma$, the same values as the MV model. This observation was used to strengthen the relation between both models. In Fig. 4 we present the results of the scaling analysis of $\chi^{\text {peak }}(N)$ for the CVB model. For $\gamma<$ $5 / 2$, the exponent $\delta$ seems to be constant and approximately equal to $0.84(2)$. For $\gamma>5 / 2$, the exponents are smaller, taking the value $\delta=0.80(2)$ for $\gamma=2.75 \delta=0.79(2)$ for $\gamma=3.00$, and $\delta=0.77$ (1) for $\gamma=3.50$. This last result is compatible with the mean-field value obtained for the MV model, $\delta_{\mathrm{MF}}=3 / 4$ [23]. The exponents for $5 / 2<\gamma<3$ appear constant and approximately equal to 0.80 , but this observation could be a finite-size effect.

Interestingly however, and despite the coincidence for large $\gamma$ values, we observe that the exponents obtained for the CBV at small $\gamma$ are different from those arising in the Vicsek model. In particular, from Ref. [17], we have for the Vicsek model $\delta=0.57$ for $\gamma=2.10$, clearly incompatible with the value obtained here. This observation prompts the conclusion that, while the Vicsek and CBV models show an analogous scaling of the threshold as a function of $\gamma$, they do not share the same critical exponents in scale-free networks.

\section{HETEROGENEOUS MEAN-FIELD ANALYSIS}

Even though the model defined in the previous section is simpler in essence than the original Vicsek model in networks, it still cannot be solved analytically. To be able to get some insight, we perform a further simplification based upon the observation in [29] that the actual shape of the function $G(u)$ is irrelevant, not even its lack of continuity at $u=0$. Inspired by this observation, we consider here a modified CBV model in which the modulating function $G(u)$ is a simple majority rule:

$$
G(u)=\operatorname{sgn}(\mathrm{u})=\left\{\begin{array}{ll}
+1 & \text { if } u>0 \\
-1 & \text { if } u<0
\end{array} .\right.
$$

With this definition, the dynamics amounts to computing the average velocity of the nearest neighbors, keeping its sign, and adding a noise term.

We can recast the dynamics of this modified CBV model in terms of a set of $d u a l$ variables $u_{i}^{*}(t)$ as

$$
\begin{gathered}
u_{i}^{*}(t+1)=G\left[\left\{u_{i}(t)\right\}\right], \\
u_{i}(t+1)=u_{i}^{*}(t+1)+\eta \xi_{i},
\end{gathered}
$$

where $\left\{u_{i}(t)\right\}$ denotes the set of possible values of $u_{i}, i \in$ $[1, N]$. From this prescription, it is easy to see by direct substitution of Eq. (9) into Eq. (8) that the dual variables $u_{i}^{*}$ fulfill the dynamic update

$$
u_{i}^{*}(t+1)=G\left[\left\{u_{i}^{*}(t)+\eta \xi_{i}\right\}\right] .
$$

The difference of this prescription with respect to Eq. (3) is that, in the original case, the final velocities are a real number, while in the modified expression the dual velocities $u_{i}^{*}(t)$ are binary variables, restricted to be $\{-1,+1\}$. From the dual variables, the original velocity values can be obtained as

$$
u_{i}(t)=u_{i}^{*}(t)+\eta \xi_{i} .
$$

Equation (10) can be further simplified in the case of a function $G(u)$ given by the sgn function. In this case, the normalization factor $k_{i}^{-1}$ in Eq. (3) can be omitted, since it does not affect the sign of the argument, so we have the final dynamical rule

$$
u_{i}^{*}(t+1)=G\left[\sum_{j} a_{i j} u_{j}^{*}(t)+\eta \sum_{j} a_{i j} \xi_{j}\right] .
$$

With the new prescription in Eq. (12), an order parameter $\phi^{*}(\eta)$ can be computed over the dual velocities $u_{i}^{*}(t)$. Since the noise $\xi_{i}$ has zero mean, the order parameters $\phi^{*}(\eta)$ and $\phi(\eta)$ coincide in the thermodynamic limit, and therefore the critical properties of the model can be computed from any prescription. In the following, we will consider the dual dynamics in Eq. (12), neglecting the star superindex to simplify notation.

We can solve the modified CBV model with the prescription given in Eq. (12) at the level of the dual variables by applying a HMF approach $[26,27,36]$ inspired by the resolution of similar spin models in networks [24,25,28,37]. To proceed, let us define $\rho_{k}(t)$ the probability that a randomly chosen node of degree $k$ is in state +1 at time $t$, and $\psi_{k}(t)$ as the probability that a node of degree $k$ flips to the state +1 at time $t$. Notice that, given Eq. (12), the probability $\psi_{k}(t)$ is independent of the state of the node $k$ considered and depends only on the state of its nearest neighbors. These two quantities are related by the rate equation

$$
\begin{aligned}
\dot{\rho}_{k}(t) & =-\rho_{k}(t)\left[1-\psi_{k}(t)\right]+\left[1-\rho_{k}(t)\right] \psi_{k}(t) \\
& =-\rho_{k}(t)+\psi_{k}(t),
\end{aligned}
$$

which in the steady state $\dot{\rho}_{k}(t)=0$ leads to

$$
\rho_{k}=\psi_{k} .
$$


To compute $\psi_{k}$, we consider the process defined by the dual variables: A node $i$ of degree $k$ looks at its $k$ nearest neighbors and considers their values $\{-1,+1\}$, plus the addition of a random number $\eta \xi_{j}$, to compute the quantity

$$
S_{i}(k)=\sum_{j} a_{i j} u_{j}+\sum_{j} \eta a_{i j} \xi_{j} .
$$

Adopting the annealed network approximation [27] to estimate this quantity in the steady state, we define the probability $Q$ that an edge departing from any node arrives at a node in state +1 . In uncorrelated networks $[38,39]$, we have

$$
Q=\frac{1}{\langle k\rangle} \sum_{k} k P(k) \rho_{k}
$$

The sum $S_{i}(k)$ can be decomposed in the contribution of the velocities of the nearest neighbors, $S_{n n}=\sum_{j} a_{i j} u_{j}$, and the noise term, $S_{\eta}=\eta \sum_{j} a_{i j} \xi_{j}$. $S_{n n}$ results from the addition of the values +1 or -1 of $k$ neighbors. Of these $k$ neighbors, there are $n$ in state +1 with probability

$$
P(n)=\left(\begin{array}{l}
k \\
n
\end{array}\right) Q^{n}(1-Q)^{k-n} .
$$

Thus,

$$
S_{n n}(n)=(+1) \times n+(-1) \times(k-n)=2 n-k,
$$

with probability $P(n)$, for $n=0,1, \ldots, k$.

In turn $S_{\eta}$ is the result of the addition of $k$ random numbers. Assuming that the noise values $\xi_{j}$ are independent random variables, and neglecting correlations between $S_{\eta}$ in different nodes, we can apply the central limit theorem and consider that $S_{\eta}$ is a Gaussian random variable of zero mean and variance $k \sigma^{2}$, where $\sigma^{2}=\eta^{2} / 12$ is the variance of the original uniform noise distribution in the interval $[-\eta / 2, \eta / 2]$. Therefore, we have that $S_{\eta}=r \in[-\infty, \infty]$ with probability

$$
P(r)=\frac{1}{\sqrt{2 \pi k \sigma^{2}}} e^{-r^{2} /\left(2 k \sigma^{2}\right)} .
$$

Applying the dynamical rule (12), the probability that the variable $u_{i}$ is flipped to +1 is equal to the probability that the sum $S_{i}(k)$ is larger than zero. This happens for $r>k-2 n$, which takes place with probability

$$
\begin{aligned}
\psi_{k} & =\sum_{n=0}^{k}\left(\begin{array}{l}
k \\
n
\end{array}\right) Q^{n}(1-Q)^{k-n} \int_{k-2 n}^{\infty} \frac{1}{\sqrt{2 \pi k \sigma^{2}}} e^{-r^{2} /\left(2 k \sigma^{2}\right)} d r \\
& =\sum_{n=0}^{k}\left(\begin{array}{l}
k \\
n
\end{array}\right) Q^{n}(1-Q)^{k-n}\left[\frac{1}{2}-\operatorname{erf}\left(\frac{k-2 n}{\sqrt{2 k} \sigma}\right)\right]
\end{aligned}
$$

where $\operatorname{erf}(z)$ is the error function [40]. Equation (21) can be simplified using the Gaussian approximation for the binomial distribution, valid when $k$ is large. Thus, the binomial with parameters $k$ and $Q$ can be approximated by a normal with mean $\mu_{b}=k Q$ and variance $\sigma_{b}^{2}=k Q(1-Q)$. Replacing the summation by an integral in Eq. (21), we have

$$
\begin{aligned}
\psi_{k} & =\frac{1}{2}-\frac{1}{\sqrt{2 \pi \sigma_{b}^{2}}} \int_{-\infty}^{\infty} e^{-\left(\mu_{b}-n\right)^{2} /\left(2 \sigma_{b}^{2}\right)} \operatorname{erf}\left(\frac{k-2 n}{\sqrt{2 k} \sigma}\right) d n \\
& =\frac{1}{2}+\operatorname{erf}\left(\frac{k y}{\sqrt{2} \sigma_{b} \sqrt{1+\frac{k \sigma^{2}}{4 \sigma_{b}^{2}}}}\right)
\end{aligned}
$$

where we have defined the new variable $y=1 / 2-Q$ that maps the disordered state, corresponding to $Q=1 / 2$, into $y=0$. Close to the disordered state, the limit of small $y$ leads to $\sigma_{b}=\sqrt{k}\left(1-4 y^{2}\right)^{-1 / 2} / 2 \simeq \sqrt{k} / 2$. Inserting this approximation into Eq. (22), we finally have

$$
\psi_{k}=\frac{1}{2}+\operatorname{erf}(\lambda \sqrt{2 k} y),
$$

where we have defined

$$
\lambda \equiv \frac{1}{\sqrt{1+\sigma^{2}}}
$$

for later convenience.

We can use Eq. (23) to self-consistently solve for $y$ in Eq. (17), considering that in the steady state $\rho_{k}=\psi_{k}$. Since $y=Q-1 / 2$, we have, from Eq. (17),

$$
y=\frac{1}{\langle k\rangle} \sum_{k} k P(k) \operatorname{erf}(\lambda \sqrt{2 k} y) \equiv \Psi(y) .
$$

For small $\lambda$, when disorder dominates, the only solution is $y=0(Q=1 / 2)$. For a sufficiently large $\lambda$, instead, a stable symmetric solution $y \neq 0$ appears. The onset of this solution takes place when

$$
\left.\frac{d \Psi(y)}{d y}\right|_{y=0}>1
$$

Performing the derivative in Eq. (25), we obtain

$$
\begin{aligned}
\left.\frac{d \Psi(y)}{d y}\right|_{y=0} & =\left.\frac{1}{\langle k\rangle} \sum_{k} k P(k) \frac{d}{d y} \operatorname{erf}(\lambda \sqrt{2 k} y)\right|_{y=0} \\
& =\frac{2 \sqrt{2}}{\sqrt{\pi}} \lambda \frac{\left\langle k^{3 / 2}\right\rangle}{\langle k\rangle}>1 .
\end{aligned}
$$

This relation defines the threshold

$$
\lambda_{c}=\frac{1}{2} \sqrt{\frac{\pi}{2}} \frac{\langle k\rangle}{\left\langle k^{3 / 2}\right\rangle},
$$

such that for $\lambda>\lambda_{c}$ there is an ordered state in the system. In terms of the noise intensity $\eta$, the ordered states takes place for $\eta<\eta_{c}$, defining the threshold

$$
\eta_{c}=\sqrt{12}\left[\frac{8}{\pi}\left(\frac{\left\langle k^{3 / 2}\right\rangle}{\langle k\rangle}\right)^{2}-1\right]^{1 / 2}
$$

For $\gamma<5 / 2$ and large $N,\left\langle k^{3 / 2}\right\rangle$ diverges, and we have $\eta_{c} \sim$ $\left\langle k^{3 / 2}\right\rangle /\langle k\rangle$, a growing function of $N$. This indicates that, in the thermodynamic limit, there is no transition, and the system is always ordered. On the other hand, for $\gamma>5 / 2,\left\langle k^{3 / 2}\right\rangle$ is finite, and there is always a transition at some finite value of $\eta_{c}$. 


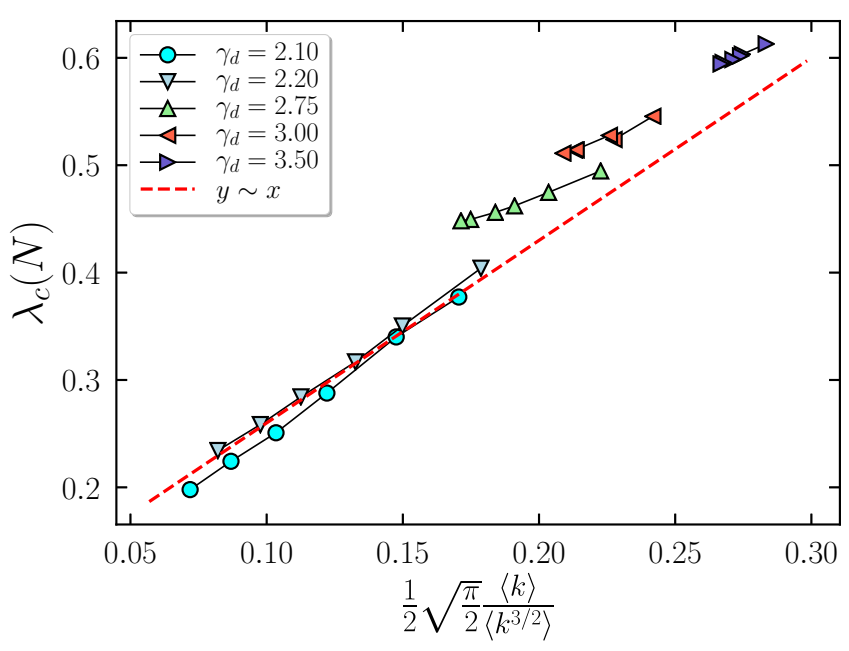

FIG. 5. Rescaled peak of the dynamic susceptibility $\lambda_{c}(N)$ as a function of the theoretical prediction, Eq. (28), in the CBV model on UCM networks of different degree exponent $\gamma$ and size $N$.

It is interesting to note that the threshold for the MV model in networks, Eq. (1), can be written as

$$
f_{c}=\frac{1}{2}-\lambda_{c}
$$

reflecting an evident relation between the MV and modified CBV models. This relation seems natural, given the equations defining the MV and modified CBV models. However, we have not been able to find a microscopic mapping between the two models.

We can compare the theoretical prediction of the modified CBV model against the results of computer simulations of the original CBV model presented in Sec. III. The HMF analysis suggests that the natural scaling of the CBV dynamics is given in terms of the $\lambda$ parameter, defined as [see Eq. (24)]

$$
\lambda(\eta)=\frac{1}{\sqrt{1+\eta^{2} / 12}} .
$$

In Fig. 5 we present a plot of the parameter $\lambda$ at criticality, $\lambda_{c}(N)=\left[1+\eta_{c}(N)^{2} / 12\right]^{-1 / 2}$, as a function of the theoretical prediction $\lambda_{c}=\frac{1}{2} \sqrt{\frac{\pi}{2}} \frac{\langle k\rangle}{\left\langle k^{3 / 2}\right\rangle}$, estimated from a direct numerical evaluation of the moments $\langle k\rangle$ and $\left\langle k^{3 / 2}\right\rangle$ in the networks considered. As we can see from this figure, while the theoretical prediction for the modified model provides a very good description of the correct scaling of the critical point for $\gamma<5 / 2$, it is still off regarding the slope by a factor around 1.7. For $\gamma>5 / 2$ the prediction is not very accurate for the system sizes considered, but it nevertheless hints towards a linear behavior for larger $N$.

We can also compute the $\beta$ exponent in the ordered phase of the modified CBV model within the HMF approximation. The order parameter for the dual variables, $\phi(\eta)=\frac{1}{N} \sum_{i} u_{i}^{*}$, can be written, assuming the steady-state condition $\rho_{k}=\psi_{k}$, as $\phi(\eta)=\sum_{k} P(k)\left[2 \psi_{k}-1\right]$. Using the expression for $\psi_{k}$ in Eq. (23), we have

$$
\phi(\eta)=2 \sum_{k} P(k) \operatorname{erf}(\lambda \sqrt{2 k} y) .
$$

Within the continuous degree approximation, replacing summations by integrals, and assuming $P(k)=(\gamma-1) m^{\gamma-1} k^{-\gamma}$, where the degree $k$ extends from the minimum value $m$ up to infinity, the equations to solve can be written as

$$
\begin{aligned}
& y=I(\gamma-1, y), \\
& \phi(\eta)=2 I(\gamma, y),
\end{aligned}
$$

where we have defined

$$
\begin{aligned}
I(\alpha, y) & \equiv(\alpha-1) m^{\alpha-1} \int_{m}^{\infty} k^{-\alpha} \operatorname{erf}(\lambda \sqrt{2 k} y) d k \\
& =\operatorname{erf}(z)+\frac{z^{2(\alpha-1)}}{\sqrt{\pi}} \Gamma\left(\frac{3}{2}-\alpha, z^{2}\right),
\end{aligned}
$$

where $z=\lambda \sqrt{2 m} y$, and $\Gamma(a, z)$ is the incomplete $\Gamma$ function [40]. Expanding to lowest order the error and incomplete $\Gamma$ functions for small $z$ we have

$$
\begin{aligned}
\sqrt{\pi} I(\alpha)= & \frac{4(\alpha-1)}{2 \alpha-3} z-\frac{4(\alpha-1)}{3(2 \alpha-5)} z^{3} \\
& +\Gamma\left(\frac{3}{2}-\alpha\right) z^{2(\alpha-1)}+O\left(z^{4}\right),
\end{aligned}
$$

where $\Gamma(a)$ is the $\Gamma$ function [40].

Since $\gamma>2,2(\gamma-1)>2$, and therefore the lowest-order term for the order parameter is

$$
\phi(\eta)=2 I(\gamma, y) \simeq \frac{8(\gamma-1)}{2 \gamma-3} \frac{z}{\sqrt{\pi}}=\frac{8(\gamma-1)}{2 \gamma-3} \lambda \sqrt{\frac{2 m}{\pi} y},
$$

linear in the parameter $y$. To find the value of $y$ we have to solve the self-consistent equation $y=I(\gamma-1, y)$, which, close to $y=0$, can be written as

$$
\sqrt{\pi} y \simeq \frac{4(\gamma-2)}{2 \gamma-5} z-\frac{4(\gamma-2)}{3(2 \gamma-7)} z^{3}+\Gamma\left(\frac{5}{2}-\gamma\right) z^{2(\gamma-2)}
$$

The solution of this equation depends on the value of $\gamma$ :

$\gamma>\frac{7}{2}$ : In this range, the leading behavior is

$$
\sqrt{\pi} y \simeq \frac{4(\gamma-2)}{2 \gamma-5} z-\frac{4(\gamma-2)}{3(2 \gamma-7)} z^{3}
$$

whose nonzero solution is

$$
y^{2} \simeq \frac{3(2 \gamma-7)}{2 m \lambda^{3}(2 \gamma-5)}\left(\lambda-\lambda_{c}\right)
$$

where

$$
\lambda_{c}=\sqrt{\frac{\pi}{2 m}} \frac{2 \gamma-5}{4(\gamma-2)},
$$

in agreement with Eq. (28) in the continuous degree approximation. From Eqs. (41) and (38) we have

$$
\phi(\eta) \sim\left(\lambda-\lambda_{c}\right)^{1 / 2} \text {. }
$$

$\frac{5}{2}<\gamma<\frac{7}{2}$ : Here, the leading behavior in the equation for $y$ is

$$
\sqrt{\pi} y \simeq \frac{4(\gamma-2)}{2 \gamma-5} z-\frac{2 \Gamma\left(\frac{7}{2}-\gamma\right)}{2 \gamma-5} z^{2(\gamma-2)}
$$


TABLE I. Summary of critical properties at the HMF level of the modified CBV model on scale-free networks.

\begin{tabular}{lcc}
\hline \hline$\gamma$ & $\lambda_{c}$ & $\beta$ \\
\hline$\gamma<\frac{5}{2}$ & 0 & $\frac{1}{5-2 \gamma}$ \\
$\frac{5}{2}<\gamma<\frac{7}{2}$ & $\sqrt{\frac{\pi}{2 m}} \frac{2 \gamma-5}{4(\gamma-2)}$ & $\frac{1}{2 \gamma-5}$ \\
$\frac{7}{2}<\gamma$ & $\sqrt{\frac{\pi}{2 m}} \frac{2 \gamma-5}{4(\gamma-2)}$ & $\frac{1}{2}$ \\
\hline \hline
\end{tabular}

The nonzero solution is, in this case,

$$
(\lambda \sqrt{2 m} y)^{2 \gamma-5} \simeq \frac{4(\gamma-2)}{2 \lambda \Gamma\left(\frac{7}{2}-\gamma\right)}\left(\lambda-\lambda_{c}\right),
$$

with $\lambda_{c}$ given by Eq. (42). This implies the scaling of the order parameter

$$
\phi(\eta) \sim\left(\lambda-\lambda_{c}\right)^{1 /(2 \gamma-5)} .
$$

$\gamma<\frac{5}{2}$ : The leading behavior corresponds to the nonzero solution

$$
y^{5-2 \gamma} \simeq \frac{\Gamma\left(\frac{5}{2}-\gamma\right)}{(2 m)^{2-\gamma} \sqrt{\pi}} \lambda^{2 \gamma-2},
$$

which corresponds to an order parameter

$$
\phi(\eta) \sim \lambda^{1 /(5-2 \gamma)} .
$$

In this case, the critical threshold is $\lambda_{c}=0$, in agreement with Eq. (28) in the infinite network limit, and corresponding to a noise threshold $\eta_{c} \rightarrow \infty$.

The critical properties of the modified CBV model at the HMF level are summarized in Table I. Interestingly, these exponents differ from those found in Ref. [25] for the MV, namely, $\beta=1 /(2 \gamma-3)$ for $3 / 2<\gamma<5 / 2$ and $\beta=1 / 2$ for $\gamma>5 / 2$.

In any case, these $\beta$ exponents do not seem to capture the behavior of the original CBV model on networks. In the case $\gamma<5 / 2$, where the critical point in the thermodynamic limits is $\lambda_{c}=0$ (equivalently $\eta_{c} \rightarrow \infty$ ), we should expect an order parameter decaying as a power law $\phi(\eta) \sim \lambda^{\beta} \sim$ $\eta^{-\beta}$ for sufficiently large $\eta$. This behavior is hampered by the fact that the effective critical point $\eta_{c}(N)$ increases very slowly with $N$; see Fig. 3 . With the system sizes considered here, we cannot observe the predicted behavior. In the case $\gamma>5 / 2$, the theory predicts a finite threshold, so we can estimate the value of the $\beta$ exponent by performing a linear regression to the form $\phi(\eta) \sim\left(\lambda-\lambda_{c}\right)^{\beta}$, where $\lambda_{c}$ is obtained from the finite-size scaling extrapolation to infinite network size. In the analysis performed in Fig. 6 we obtain a very good fit to this expression, with an exponent $\beta \simeq 1 / 2$, apparently independent of the degree exponent $\gamma$. This numerical value of the $\beta$ exponent is equal to the pure mean-field prediction corresponding to $\gamma>7 / 2$, in agreement with the result obtained for $\gamma=3.50$. For $\gamma=3.00$ and $\gamma=2.75$, our numerical results are in strong disagreement with the HMF prediction, which in this case would correspond to an exponent larger than 1. The failure of HMF to recover the numerical observation can be associated with different sources of error. First, the failure could also be attributed to the difference between the numerically considered CBV model

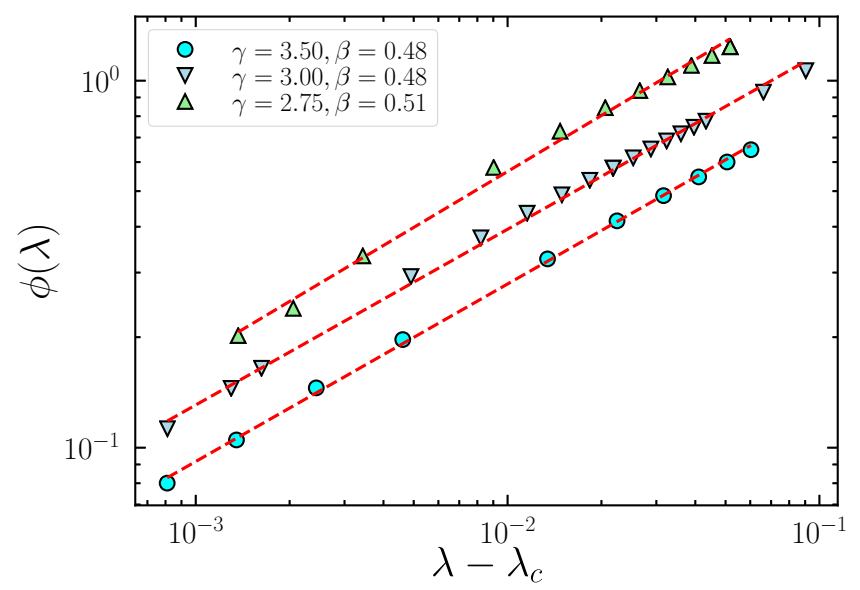

FIG. 6. Plot of the order parameter as a function of the rescaled control parameter $\lambda$ in the vicinity of the transition. The $\beta$ values quoted are obtained by means of a linear regression to the form $\phi(\lambda) \simeq\left(\lambda-\lambda_{c}\right)^{\beta}$, with $\lambda_{c}$ equal to the finite-size scaling extrapolation in the thermodynamic limit. Plots have been shifted vertically for the sake of clarity.

and the modified version actually solved. The effect of a modulating $G(u)$ function that provides a binary output could be enough to change the critical exponents of this flocking model. Also, it could be due to an intrinsic failure of HMF, as observed in other dynamical processes, particularly epidemic spreading on scale-free networks [36].

\section{DISCUSSION}

In this paper we have contributed to the study of the effects of heterogeneous social interactions in the behavior of flocking dynamics. To this end, we have studied the dynamics of the flocking model proposed by Czirók, Barabási, and Vicsek (CBV), in which the interactions leading to the alignment of velocity are mediated by a scale-free network, as observed in several animal social contact networks. In opposition to the classical Vicsek model, in which velocity is a vector, the CBV model considers a scalar velocity. Despite this difference, both Vicsek and CBV models show an analogous behavior in power-law networks with degree distribution $P(k) \sim k^{-\gamma}$ : For mildly heterogeneous networks, with $\gamma>5 / 2$, the models show a true order-disorder transition, located at a finite value of a noise intensity control parameter $\eta$. On the other hand, for highly heterogeneous networks, with $\gamma<5 / 2$, the transition is absent in the thermodynamic limit, the systems being ordered for all physical values of the noise intensity. Interestingly, despite the coincidence in the position of the critical point, the Vicsek and CBV models show different critical exponents ruling the growth of the susceptibility at the transition.

In order to gain insight into this phenomenology, we consider a modification of the CBV model that can be solved within an heterogeneous mean-field (HFM) approximation, typical for the analysis of dynamical processes on networks. In our analysis, we confirm the results obtained numerically: The critical noise in the modified CBV model scales as the degree moment $\left\langle k^{3 / 2}\right\rangle$ and thus experiences a transition between 
a finite and an infinite value, in the thermodynamic limit, when $\gamma$ crosses the boundary 5/2. The analytical expression for the threshold is compared with numerical values, showing a reasonably good fit. The HMF approximation allows us also to compute the exponent $\beta$ characterizing the ordered phase. The values obtained, however, are not reproduced by numerical simulations. This failure of HFM can be attributed to either insufficiently large network sizes, which do not allow us to explore deep enough into the critical phase, or to an intrinsic deficiency of the HMF approximation, already observed in other dynamical processes.

Our results contribute to deepen the understanding of the dynamics of flocking mediated by social interactions, with the introduction of a model amenable to a simple analytical characterization and that allows for more complex extensions, such as the introduction of weights in the social ties. The analysis of these kind of models can be improved, beyond the simplest HMF approximation, by considering the full structure of the network in a quenched mean-field approach [41,42]. Finally, the observation of a crossover behavior at a degree exponents $\gamma=5 / 2$ might be conjectured to be a general feature of flocking models based on velocity averaging. Further investigation of this issue should be devoted to validate this conjecture. Additionally, one can envisage potential applications in situations where what really matters is not the particular direction of motion of a given animal, but its dynamic response to particular stimuli that can be regarded as a scalar quantity transmitted through a network of social contacts. We can think of situations, such as in the presence of predators, where assessing the existence of movement might be more important than establishing an average direction of motion. The results presented here would then have implications regarding the ability of the system to respond collectively as a function of the noise threshold.

\section{ACKNOWLEDGMENTS}

We acknowledge financial support from the Spanish MINECO, under Projects No. FIS2016-76830-C2-1-P and No. FIS2016-76830-C2-2-P. R.P.-S. acknowledges additional financial support from ICREA Academia, funded by the Generalitat de Catalunya.
[1] T. Vicsek and A. Zafeiris, Phys. Rep. 517, 71 (2012).

[2] S. Ramaswamy, Annu. Rev. Condens. Matter Phys. 1, 323 (2010).

[3] A. Cavagna, I. Giardina, and T. S. Grigera, Phys. Rep. 728, 1 (2018).

[4] C. W. Reynolds, SIGGRAPH Comput. Graph. 21, 25 (1987).

[5] T. Vicsek, A. Czirok, E. Ben-Jacob, I. Cohen, and O. Shochet, Phys. Rev. Lett. 75, 1226 (1995).

[6] V. Méndez, D. Campos, and F. Bartumeus, Stochastic Foundations in Movement Ecology (Springer Verlag, Berlin, 2014).

[7] D. J. T. Sumpter, Philos. Trans. R. Soc. London B 361, 5 (2006).

[8] D. J. Sumpter, Collective Animal Behavior (Princeton University Press, Princeton, 2010).

[9] F. Ginelli, Eur. Phys. J. Spec. Top. 225, 2099 (2016).

[10] F. Ginelli and H. Chaté, Phys. Rev. Lett. 105, 168103 (2010).

[11] J. Toner and Y. Tu, Phys. Rev. Lett. 75, 4326 (1995).

[12] H. Ling, G. E. Mclvor, K. van der Vaart, R. T. Vaughan, A. Thornton, and N. T. Ouellette, Nat. Ecol. Evol. 3, 943 (2019).

[13] M. Newman, Networks: An Introduction (Oxford University Press, New York, 2010).

[14] N. W. Bode, A. J. Wood, and D. W. Franks, Anim. Behav. 82, 29 (2011).

[15] N. W. F. Bode, A. J. Wood, and D. W. Franks, Behav. Ecol. Sociobiol. 65, 117 (2011).

[16] A. Sekunda, M. Komareji, and R. Bouffanais, Netw. Sci. 4, 244 (2016).

[17] M.-C. Miguel, J. T. Parley, and R. Pastor-Satorras, Phys. Rev. Lett. 120, 068303 (2018).

[18] A.-L. Barabási and R. Albert, Science 286, 509 (1999).

[19] D. Croft, R. James, and J. Krause, Exploring Animal Social Networks (Princeton University Press, Princeton, 2008).

[20] D. Lusseau, Proc. R. Soc. B 270, S186 (2003).

[21] T. G. Manno, Animal Behav. 75, 1221 (2008).

[22] M. J. de Oliveira, J. Stat. Phys. 66, 273 (1992).

[23] L. F. C. Pereira and F. G. Brady Moreira, Phys. Rev. E 71, 016123 (2005).
[24] C. Huepe and M. Aldana-González, J. Stat. Phys. 108, 527 (2002).

[25] M. Aldana and H. Larralde, Phys. Rev. E 70, 066130 (2004).

[26] R. Pastor-Satorras and A. Vespignani, Phys. Rev. Lett. 86, 3200 (2001).

[27] S. N. Dorogovtsev, A. V. Goltsev, and J. F. F. Mendes, Rev. Mod. Phys. 80, 1275 (2008).

[28] H. Chen, C. Shen, G. He, H. Zhang, and Z. Hou, Phys. Rev. E 91, 022816 (2015).

[29] A. Czirók, A.-L. Barabási, and T. Vicsek, Phys. Rev. Lett. 82, 209 (1999).

[30] J. Buhl, D. J. T. Sumpter, I. D. Couzin, J. J. Hale, E. Despland, E. R. Miller, and S. J. Simpson, Science 312, 1402 (2006).

[31] G. Ariel and A. Ayali, PLoS Comput. Biol. 11, e1004522 (2015).

[32] M. Catanzaro, M. Boguñá, and R. Pastor-Satorras, Phys. Rev. E 71, 027103 (2005).

[33] S. C. Ferreira, C. Castellano, and R. Pastor-Satorras, Phys. Rev. E 86, 041125 (2012).

[34] C. Castellano and R. Pastor-Satorras, Eur. Phys. J. B 89, 243 (2016)

[35] J. L. Cardy (Ed.), Finite Size Scaling, Current Physics-Sources and Comments Vol. 2 (North-Holland, Amsterdam, 1988).

[36] R. Pastor-Satorras, C. Castellano, P. Van Mieghem, and A. Vespignani, Rev. Mod. Phys. 87, 925 (2015).

[37] C. Castellano and R. Pastor-Satorras, J. Stat. Mech.: Theory Exp. (2006) P05001.

[38] R. Pastor-Satorras, A. Vázquez, and A. Vespignani, Phys. Rev. Lett. 87, 258701 (2001).

[39] M. E. J. Newman, Phys. Rev. Lett. 89, 208701 (2002).

[40] M. Abramowitz and I. A. Stegun, Handbook of Mathematical Functions (Dover, New York, 1972).

[41] C. Castellano and R. Pastor-Satorras, Phys. Rev. Lett. 105, 218701 (2010).

[42] F. Huang, H. Chen, and C. Shen, Europhys. Lett. 120, 18003 (2017). 